\title{
Simulation and Noise Analysis of Multimedia Transmission in Optical CDMA Computer Networks
}

\author{
Nasaruddin $^{1} \&$ Tetsuo Tsujioka ${ }^{2}$ \\ ${ }^{1}$ Electrical Engineering Department, Faculty of Engineering, Syiah Kuala University \\ Email: nasaruddin@ unsyiah.net \\ ${ }^{2}$ Graduate School of Engineering, Osaka City University \\ Email: tsujioka-H22@info.eng.osaka-cu.ac.jp
}

\begin{abstract}
This paper simulates and analyzes noise of multimedia transmission in a flexible optical code division multiple access (OCDMA) computer network with different quality of service (QoS) requirements. To achieve multimedia transmission in OCDMA, we have proposed strict variable-weight optical orthogonal codes (VW-OOCs), which can guarantee the smallest correlation value of one by the optimal design. In developing multimedia transmission for computer network, a simulation tool is essential in analyzing the effectiveness of various transmissions of services. In this paper, implementation models are proposed to analyze the multimedia transmission in the representative of OCDMA computer networks by using MATLAB simulink tools. Simulation results of the models are discussed including spectrums outputs of transmitted signals, superimposed signals, received signals, and eye diagrams with and without noise. Using the proposed models, multimedia OCDMA computer network using the strict VW-OOC is practically evaluated. Furthermore, system performance is also evaluated by considering avalanche photodiode (APD) noise and thermal noise. The results show that the system performance depends on code weight, received laser power, APD noise, and thermal noise which should be considered as important parameters to design and implement multimedia transmission in OCDMA computer networks.
\end{abstract}

Keywords: computer networks; multimedia transmission; Optical CDMA.

\section{Introduction}

In the future, computer networks have a demand for transmitting large amounts of data between many computers, which are all connected in the same network. They should be able to accommodate the growing numbers of users and to deliver multimedia services (e.g., Internet telephony, real-time image sharing, data transfer, video, and voice) at the same time. One of the solutions is optical fiber, which has distinct advantages over other transmission media because it has extremely large bandwidth. With forecasts of Internet traffic increasing rapidly, optical fiber is the most appropriate multimedia transmission medium to carry the bulk of future network traffic. In general, there are three possible ways to share bandwidth for a number of connections, namely optical time- 
division multiple-access (OTDMA) [1], wavelength division multiplexing (WDM) [2], and Optical code-division multiple-access (OCDMA) [3].

In the OTDMA technique, each user is allocated a specific time slot, where it requires short-pulsed diode lasers and provides only moderate improvements in bandwidth utilization. No partial network upgrade is possible; this makes OTDMA systems less flexible than that is desirable. While the WDM technique divides the available optical bandwidth into distinct wavelength channels that are used concurrently by different users. To increase the number of channels in the system, narrower bandwidth slots could be required, which would put further restrictions on the performance of the laser sources and other optical components. Furthermore, both OTDMA and WDM techniques require deterministic wavelength assignment and strict synchronous time-slot control, respectively. On the other hand, the OCDMA is a technique in which each user is assigned a specific code rather than a specific time slot or wavelength. This allows the same available bandwidth to be shared among the users, and the code assignments can be flexible and re-configurable. Recently, the OCDMA has received much attention as a flexible multiple access technique for computer networks due to its potential for re-configurability of multi-user (codes), privacy and security in transmission, asynchronous access, simplified and decentralized network control, and the ability to support multimedia transmission with different data rates and quality-of-service (QoS) [3]. This characteristic of OCDMA makes it well-suited technology with increased capacity and large number of users of burst networks (i.e., local area network (LAN) [4] and possibly metro area network (MAN) applications [5]).

In OCDMA systems, each user (node) is assigned to a specific codeword as his/her code address. Therefore, coding scheme is a prerequisite for designing the OCDMA system. In most incoherent OCDMA systems, only unipolar $(0,1)$ codes can be used. A class of unipolar codes called optical orthogonal code (OOC) has been applied for OCDMA [6]-[9]. However, most conventional OOCs were considered with respect to identical code length and weight to satisfy code correlation properties; that is to guarantee a certain number of active equal data rates and equal error performance among users. When these OOCs are used for multimedia transmission, their correlation properties can be changed. To meet multimedia transmission requirements, therefore, we have designed strict variable-weight OOCs (VW-OOCs) [10]. However, simulation analysis and implementation models for computer networks were not demonstrated in the paper. On the other hand, implementation model, noise analysis, and the feasibility of employing strict VW-OOCs are also crucial towards practical multimedia transmission in flexible OCDMA computer networks. 
This paper proposes the feasible simulation models for multimedia transmission in OCDMA computer networks based on MATLAB Simulink tools. One crucial part of the design of multimedia transmission models for OCDMA networks is the efficient modelling by using strict VW-OOCs which support multimedia transmission in flexible OCDMA computer networks. This paper will demonstrate the developed multimedia transmission models through extensive simulations on the representative multimedia transmission in OCDMA based computer network. Simulation results of the implementation models are demonstrated and those include spectrum outputs of transmitted signals, superimposed signals, received signals, and eye-diagram. Moreover, this paper also considers theoretically performance analysis with avalanche photodiode (APD) noise and thermal noise. For convenience, the performance with and without hard-limiter are considered. The strict VW-OOCs show that a user with large code weigh performs better than a user with small code weight. This can fulfill the requirements of multimedia transmission that support different quality of service (QoS) in OCDMA computer networks.

The rest of the paper is organized as follows. Optical CDMA computer network architecture is presented in Section II. In Section III, optical CDMA coding for multimedia transmission is reviewed. Simulation models and noise analysis for multimedia transmission in OCDMA computer networks are presented in Section IV. Simulation results, analytical results and discussions are discussed in Section V. Finally, we conclude with a brief summary of results.

\section{Optical CDMA Computer Networks}

A typical OCDMA architecture for computer network (i.e., OCDMA-based LAN) is shown in Figure 1 [11]. At the physical layer, it represents a passive local network (PON) with a star topology. However, at the configuration layer, it is a broadcast and select network. Computer network needs to connect a large number of users within its area with a high speed transmission. The network offers the advantage of a random access from the user to the network. By using OCDMA technology in a computer network, it makes efficient the use of channel by providing asynchronous access to each user. Each user will be assigned to a codeword of a strict VW-OOC. The star coupler superimposes the optical signals from all nodes. Each node is connected to one input port and one output port by an optical fiber. Then, the nodes and the star coupler form a shared medium network. The input and output ports of the nodes are called transmitter and receiver, respectively. A node that is transmitting data to the other nodes on the network encodes the individual " 1 " bits into "chips" according to the strict VW-OOC. Every node in the network is assigned to a specific codeword. Transmitted signals on the inputs enter the coupler on several transmitters, where the optical power from all transmitters is merged. 
The power is then split equally among receivers. The same signal emerges on all the receivers of the coupler and is transmitted to all nodes. Furthermore, OCDMA technology enables an access platform, which provides interconnections between end-nodes and the wide area Internet through routers.

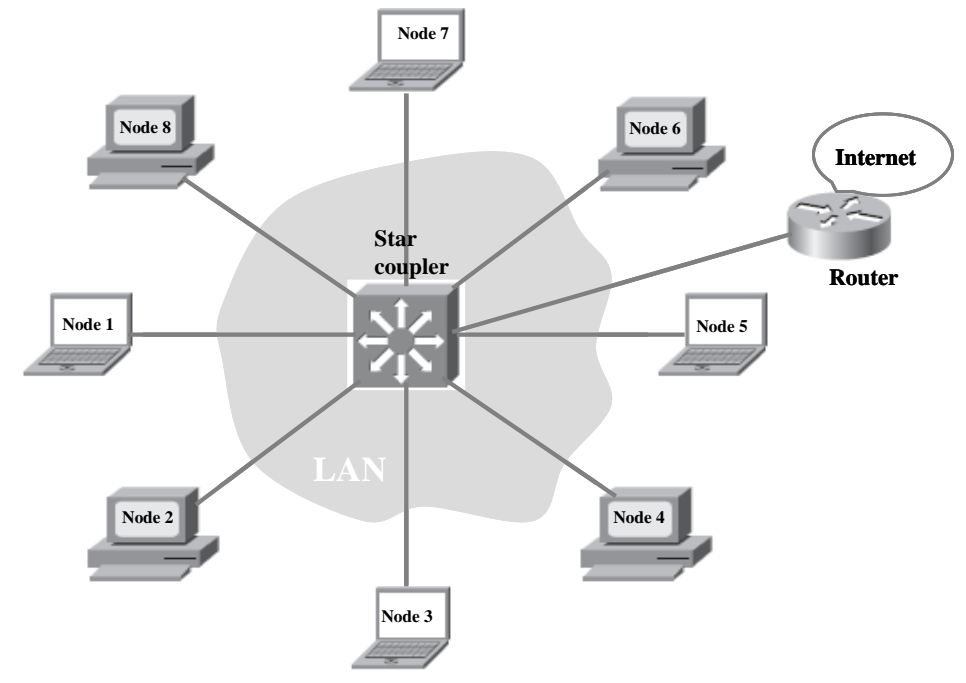

Figure 1 OCDMA-based computer network architecture.

Such network should be able to support multimedia applications with different performance levels and various QoS. By employing the strict VW-OOCs in the networks, such requirements can be met because the performance of a user depends on his code weight. The highest priority user should be assigned to the largest weight to guarantee the lowest bit error probability in the network. While the user which can tolerate some transmission errors is then assigned to the smallest weight. Therefore, by employing the strict VW-OOCs in computer network, it may allow differentiated QoS at the physical layer for multimedia transmission.

\section{Optical CDMA Coding for Multimedia Transmission}

It has been shown that multiple-length variable-weight optical orthogonal codes $[12,13]$ or strict VW-OOCs [10] can support multimedia transmission in OCDMA networks. In this paper, we will focus only on the implementation of the strict VW-OOCs for OCDMA computer network through the MATLAB simulink tools. So we here present a brief review on the strict VW-OOCs. A strict VW-OOC is a class of OOCs where all codewords are the same code length but may have different code weights. The main purpose of the strict VW- 
OOCs is that the code weight has a direct effect on the performance of an OCDMA system. A codeword with a large weight is less sensitive to interference than that with a smaller code weight.

A strict VW-OOC, $C$, is generally denoted by $(n, W, \lambda, Q)$ where $n, W, \lambda$, and $Q$ are the code length, a set of code weights $W=\left\{w_{1}, \ldots, w_{L}\right\}$, a maximum value of auto- and cross-correlation, and a set of fraction of codewords $Q=\left\{q_{1}, \ldots\right.$, $\left.q_{L}\right\}$, respectively, where $L$ is the number of different weights in a code set. The $(0,1)$ sequences of a VW-OOC are called its codewords. The total number of " $1 \mathrm{~s}$ " in each codeword defines the code weight $w_{l}$, where $w_{l}$ is $l$ th code weight and $l=1, \ldots, L$. A strict VW-OOC can also be viewed as a family of $w_{l}$-set of integer modulo $n$, in which each $w_{l}$-set corresponds to a codeword and the integers within each $w_{l}$-set specify the " $1 \mathrm{~s}$ " positions in the codeword. The cardinality of a VW-OOC, denoted by $|C|$, is the number of codewords in a code set $C$.

The strict VW-OOCs can be constructed by distinct set approach and random method. The algorithms for both methods can be found in [10]. Let us construct a $(141,\{5,4\}, 1,\{1 / 2,1 / 2\})$ strict VW-OOC based on the distinct set approach in [10]. The codewords of the code are listed in Table 1. These codewords are used in our simulation for six-user in multi-media transmission computer network. Using this code, two different services can be supported in the network; three users of weight 5 for the first service (i.e., video) and three users of weight 4 for the second service (i.e., voice).

Table $1 \quad(141,\{5,4\}, 1,\{1 / 2,1 / 2\})$ strict VW-OOC codewords.

\begin{tabular}{cc||cc}
\hline No & "1s" Positions for $\boldsymbol{w = 5}$ & No & "1s" Positions for $\boldsymbol{w = 4}$ \\
\hline 1. & $(0,1,8,25,56)$ & 4. & $(0,4,16,38)$ \\
2. & $(0,2,11,29,52)$ & 5. & $(0,5,19,45)$ \\
3. & $(0,3,13,33,70)$ & 6. & $(0,6,21,49)$ \\
\hline
\end{tabular}

To ensure that the correlation of $(141,\{5,4\}, 1,\{1 / 2,1 / 2\})$ strict VW-OOC is exactly bounded by one for overall codewords, the number of used delay elements of a codeword and among codewords is evaluated by a computer simulation. The simulated result is shown in Figure 2, where the maximum correlation value is definitely bounded by one because there is no repeated used of the delay elements in the code. Therefore, this code can guarantee the least multiple access interference (MAI) for incoherent multimedia transmission in OCDMA computer network. 


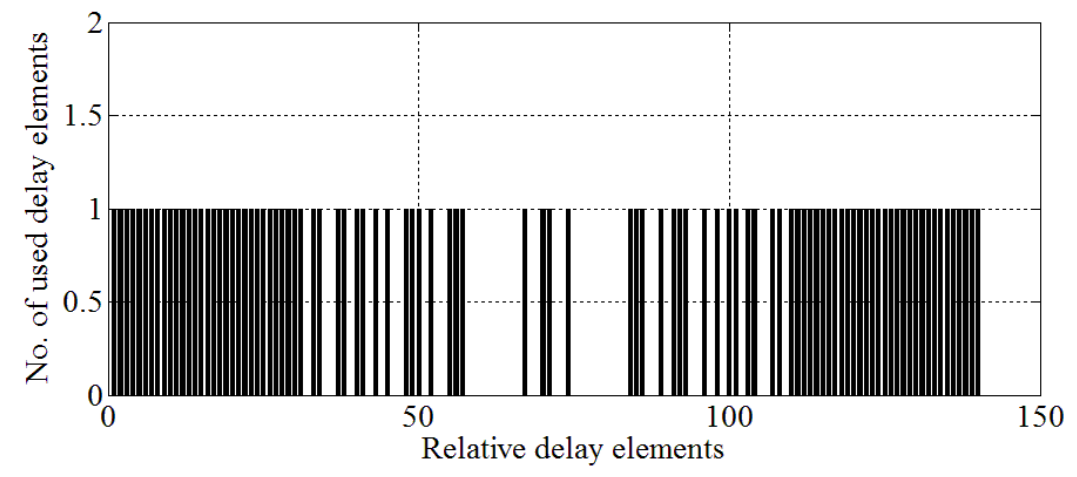

Figure 2 Relative delay elements used in a $(141,\{5,4\}, 1,\{1 / 2,1 / 2\})$ strict VW-OOC in Table 1.

\section{Simulation Model and Noise Analysis}

In this Section, we present simulation models of multimedia transmission in flexible OCDMA computer network, which is similar to but simpler than the system it represents. In the network, we consider two different weights of coding to accommodate two different services in multimedia OCDMA system. One of purposes of a MATLAB/simulink model for multimedia transmission in OCDMA computer network is to enable the engineer to implement the optical coding and the visualization of the physical principal working of the multimedia network. Since MATLAB/simulink has no function to simulate optical noise sources, we present theoretical analysis for optical noise sources. However, noise channel model is presented in our simulation model to see the effect of noise in the network.

\subsection{Simulation Set Up}

Simulation parameters are listed in Table 2. Here, $1 \mathrm{~Gb} / \mathrm{s}$ pulse laser diodes and APD receivers are assumed in the simulink model. In Figure 3, there are sixpair of users requiring six different $(0,1)$ codewords from the code set of $(141,\{5,4\}, 1,\{1 / 2,1 / 2\})$ strict VW-OOC as their addresses. However, in large capacity networks, other users can easily be accommodated by using the large number of strict VW-OOC codewords. Every user is assigned to the $(0,1)$ codeword from a strict VW-OOC. The large weight codewords with $w_{1}=5$ are assigned to the first three users (user 1-3) for high priority service (e.g., video), while the small weight codewords with $\mathrm{w}_{2}=4$ are assigned to the remaining users (user 4-6) for less priority service (e.g., voice). At transmitter side, information data for every user is generated by Bernoulli binary generator. Data at active transmitters are 
encoded with the desired codeword using an ON-OFF keying (OOK) modulation. The OOK modulation is performed by sum function of information data and user's codeword in the simulink. After encoding and modulating processes, the active transmitters superimpose their outputs over optical fiber using a star coupler (sum function in the simulink) and are then distributed to each receiver. At receiver side, decoding is matched to the desired codeword by using XCORR function. If any pair of transmitter and receiver codeword matches exactly, the output of correlator has an auto-correlation peak which recognizes that the transmitter has transmitted a "1" data bit. Otherwise, the correlator output has no peak but sum of cross-correlation between other codeword users.

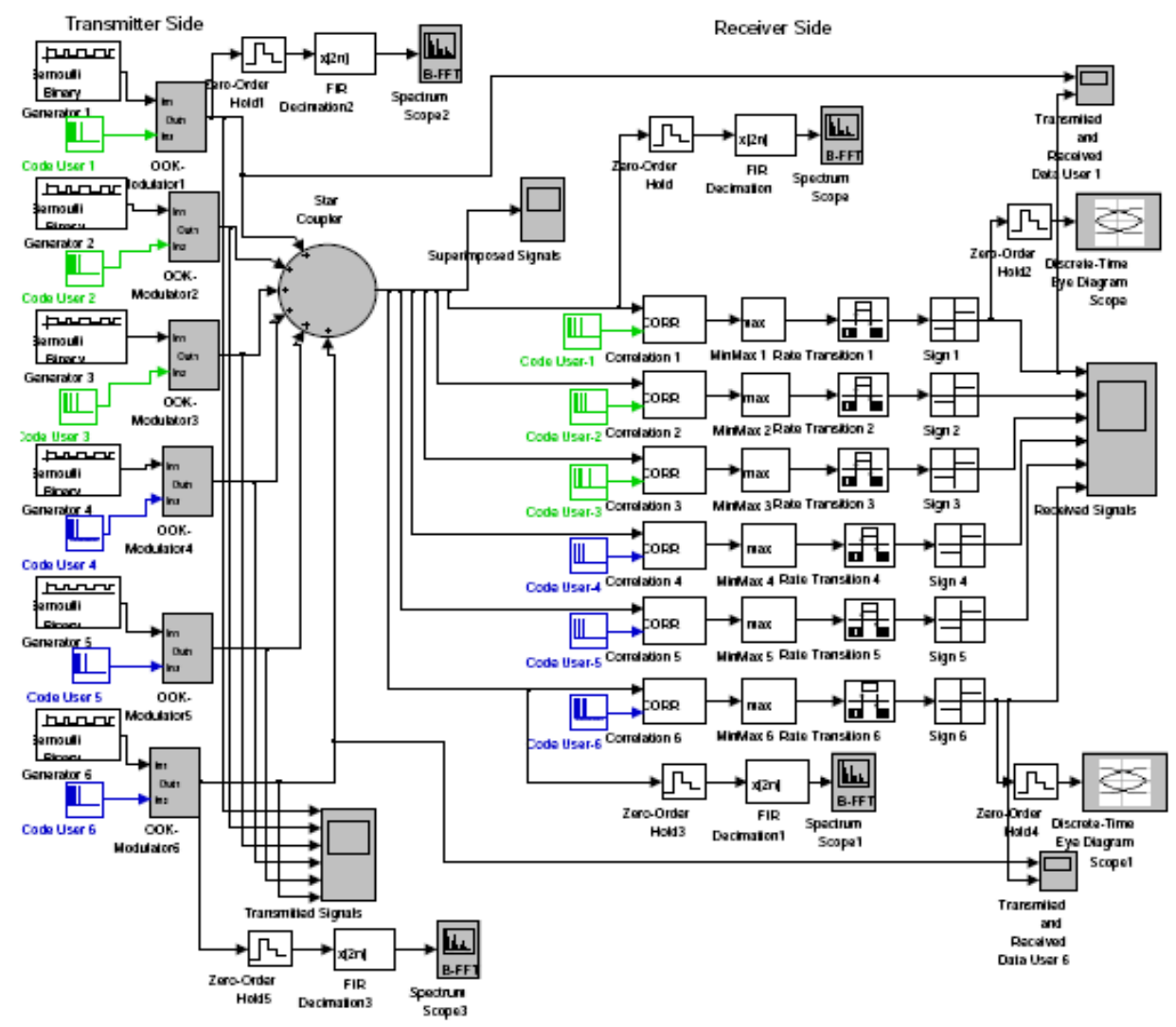

Figure 3 A simulink model for multimedia transmission in OCDMA computer network.

Figure 4 shows a noise model for multimedia transmission in OCDMA computer network. The principle working of the network is the same as in 
Figure 3 but we consider that the channel is corrupted by additive white Gaussian noise (AWGN). We placed it after star coupler to represent AWGN noise channel, because a transmission power controlled OCDMA computer network was assumed.

Table 2 Simulation Parameters.

\begin{tabular}{ll}
\hline Parameter & Constraint \\
\hline Coding Scheme & $(141,\{5,4\}, 1,\{1 / 2,1 / 2\})$ strict \\
Number of Services & 2 \\
Number pairs of users & 6 \\
Discrete step & $100 \mathrm{ps}$ \\
Chip duration & $1 \mathrm{~ns}$ \\
Bit duration & $141 \mathrm{~ns}$ \\
Data rate & $7.1 \mathrm{Mbps}$ \\
\hline
\end{tabular}

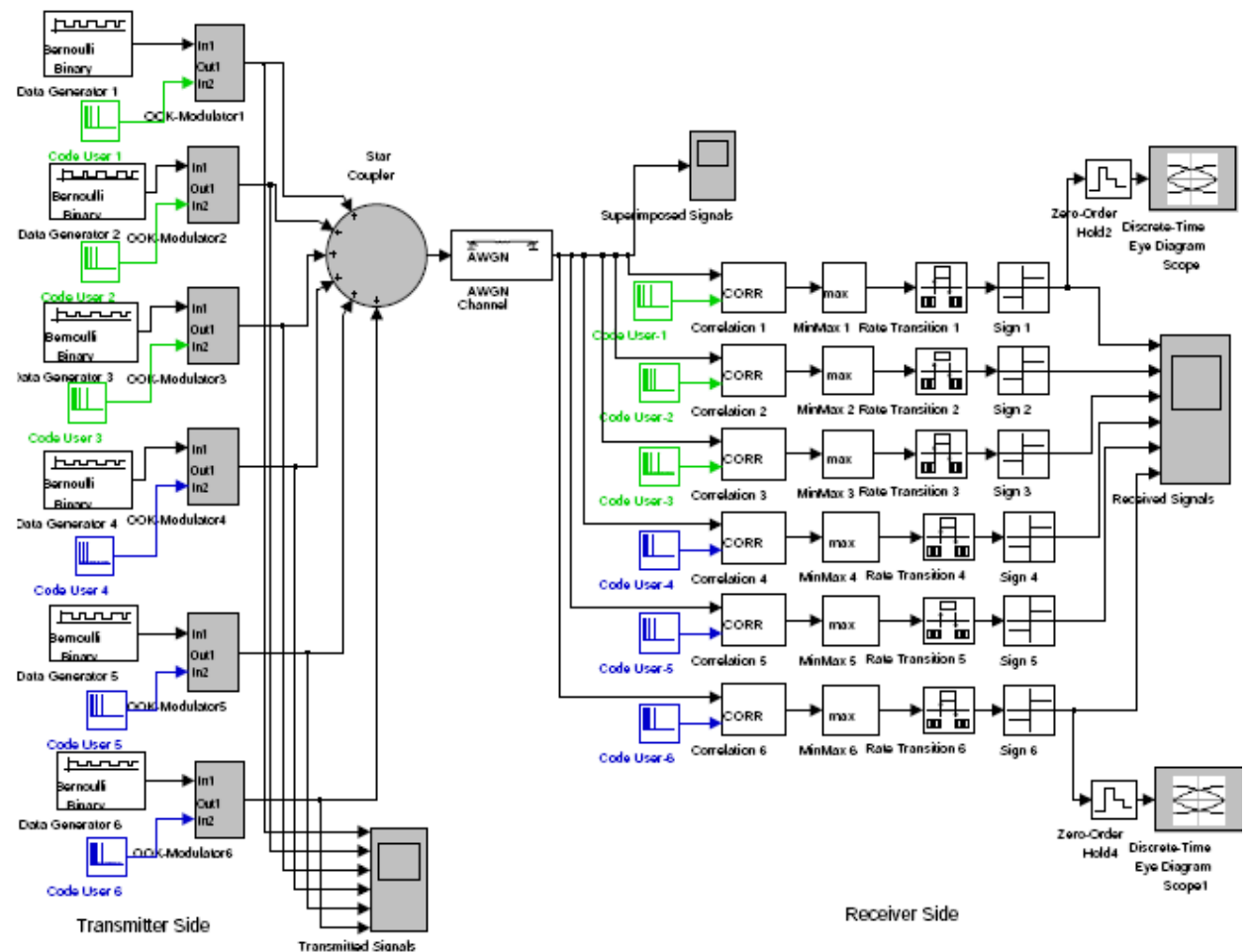

Figure 4 A simulink noise model for multimedia transmission in OCDMA computer network. 


\subsection{Noise Analysis}

The bit error probability of constant weight OOC system when using an APD and taking into account the effect of thermal noise can be found in [14]-[16]. In this paper, we analyze the bit error probability of the strict VW-OOC system by considering the effect of APD noise and thermal noise.

We assume $Y_{l}$ as correlator output in photon count for binary data's decision at the APD receiver with the $l$ th code weight $w_{l}$. First, we derive the variance $\sigma_{b, l}^{2}$ of $Y_{l}$ when binary data bit of $b \in\{0,1\}$ is transmitted. $\sigma_{b, l}^{2}$ can be expressed as

$$
\sigma_{b, l}^{2}=F G \mu_{b, l}+\sigma_{\mathrm{n}}^{2},
$$

where $F$ is the APD excess noise factor, $G$ is the average APD gain, $\mu_{b, l}$, is the mean value of $Y_{, l}$, and $\sigma_{\mathrm{n}}^{2}$ is the variance of the thermal noise within a chip interval. These parameters are given by

$$
\begin{aligned}
& F=k_{\text {eff }} G+(2-1 / G)\left(1-k_{\text {eff }}\right), \\
& \mu_{b, l}=G\left(r_{l} b w_{l}+r_{\mathrm{d}}\right), \\
& \sigma_{\mathrm{n}}^{2}=\frac{2 K_{\mathrm{B}} T^{\circ}}{e^{2} R_{\mathrm{L}}} T_{\mathrm{c}},
\end{aligned}
$$

where $k_{\text {eff }}$ is the APD effective ionization ratio, $K_{\mathrm{B}}$ is Boltzmann's constant of $1.379 \times 10^{-23} \mathrm{~J} / \mathrm{K}, T^{\mathrm{o}}$ is the receiver noise temperature, $R_{\mathrm{L}}$ is the receiver load resistance, and $e$ is the electron charge of $1.601 \times 10^{-19} \mathrm{C}$. The photon count $r_{\mathrm{d}}$ due to the APD dark current within a chip interval, the average number of absorbed photons $r_{l}$ per received single-user chip for $w_{l}$ are given by

$$
\begin{aligned}
& r_{\mathrm{d}}=\frac{I_{\mathrm{d}}}{e} T_{\mathrm{c}}, \\
& r_{l}=\frac{\eta \lambda_{0} P_{\mathrm{av}} n T_{\mathrm{c}}}{h c w_{l}^{2}},
\end{aligned}
$$

where $I_{\mathrm{d}}$ is the APD dark current, $\eta$ is the APD efficiency, $\lambda_{0}$ is the laser wavelength, $P_{\mathrm{av}}$ is the average received laser power (of a single-user), $T_{\mathrm{c}}$ is chip duration, $h$ is Plank's constant of $6.624 \times 10^{-34} \mathrm{~J}$, and $c$ is the light speed of $3 \times$ $10^{8} \mathrm{~m} / \mathrm{s}$. 
If the collected photon count in any bit interval is greater than threshold $T_{h}$, "1" is decided, and decided " 0 " otherwise. Denoting the photon count collected in one bit interval by $Y$, the bit error probability, $P_{E}$, is given by

$$
P_{E}=\frac{1}{2} \min _{T_{h}}\left(P_{r}\left\{Y \leq T_{h, l} \mid b=1\right\}+P_{r}\left\{Y>T_{h, l} \mid b=0\right\}\right),
$$

where $b$ denotes the transmitted data bit and $1 / 2$ denotes equiprobable $(1,0)$ data bit transmission. The APD output has been well modelled as Gaussian process using the central limit theorem [19] although the total number of absorbed photons is Poisson random variable [15]. Assuming continuous Gaussian approximation for the photon count $Y$, we have the expression given by

$$
P_{r}\left\{Y \leq T_{h, l} \mid b=1\right\}=Q\left(\frac{\mu_{1 l}-T_{h}}{\sqrt{\sigma_{1 l}^{2}}}\right),
$$

and

$$
\begin{aligned}
P_{r}\left\{Y>T_{h, l} \mid b=0\right\} & =P_{r}\left\{Y>T_{h, l} \mid b=0, Z=w_{l}\right\} P_{r}\left\{Z=w_{l}\right\} \\
& +P_{r}\left\{Y>T_{h, l} \mid b=0, Z \neq w_{l}\right\} P_{r}\left\{Z \neq w_{l}\right\},
\end{aligned}
$$

where the random variable $Z \in\left\{1,2, \ldots, w_{l}\right\}$ denotes the number of interfered chip positions in the interval of the desired user. In the $(n, W, \lambda, Q)$ strict VWOOC, each interfering user may contribute only to one chip overlap (hit) with the intended receiver. Then, the probability that one of nonzero positions of a user codeword with weight $w_{l}$ overlaps with one of nonzero positions of the desired codeword with weight $w_{l}$, is given by

$$
p_{l}=\frac{w_{l} w_{l^{\prime}}}{2 n}
$$

where $l, l^{\prime} \in\{1, \cdots, L\}$ and $w_{l} \neq w_{l}$. Let us assume there are $M=\sum_{l=1}^{L} M_{l}$ users in the network, where $M_{l}$ is the users with code weight $w_{l}$. Therefore, there are $M$-1 users that could interfere with the desired user. For the case without hardlimiter at the receiver, it can be evaluated as [17]

$$
\begin{aligned}
P_{r}\left\{Z=w_{l}\right\}= & \sum_{\substack{I_{1}+I_{2}+\cdots+I_{L}=T_{h, l} \\
L_{h} I_{l}}}^{M-1}\left(\begin{array}{c}
M_{l}-1 \\
I_{l}
\end{array}\right) p_{l^{\prime}}^{I_{l}}\left(1-p_{l}\right)^{M_{l}-1-I_{l}} \\
& . \prod_{\substack{l^{\prime}=1 \\
l^{\prime} \neq l}}^{L}\left(\begin{array}{c}
M_{l^{\prime}} \\
I_{l^{\prime}}
\end{array}\right) p_{l^{\prime}}^{I_{l^{\prime}}}\left(1-p_{l^{\prime}}\right)^{M_{l^{\prime}}-I_{l^{\prime}},},
\end{aligned}
$$


where $I_{l}$ is the number of the interferers with codewords $w_{l}$, and $I=\sum_{l=1}^{L} I_{l}, I \neq$ 0 . For the receiver with double hard-limiter (DHL), the signal of the desired user is immediately after the first optical hard-limiter. The bit error probability of the strict VW-OOC with DHL can be evaluated as

$$
\begin{aligned}
P_{r}\left\{Z=w_{l}\right\}= & \sum_{i=0}^{w_{l}-1}(-1)^{i}\left(\begin{array}{c}
w_{l} \\
i
\end{array}\right)\left[\left(1-\frac{p_{l} i}{w_{l}}\right)\right]^{M_{l^{\prime}}-1} \prod_{\substack{l^{\prime}=1 \\
l^{\prime} \neq l}}^{L}\left[\left(1-\frac{p_{l^{i}}}{w_{l}}\right)\right]^{M_{l^{\prime}}} \\
& +(-1)^{w_{l}}\left(1-p_{l}\right)^{M_{l^{\prime}-1}} \prod_{\substack{l^{\prime}=1 \\
l^{\prime} \neq l}}^{L}\left(1-p_{l^{\prime}}\right)^{M_{l^{\prime}}} .
\end{aligned}
$$

The remaining probabilities in (9) are calculated as

$$
P_{r}\left\{Y>T_{h, l} \mid b=0, Z=w_{l}\right\}=Q\left(\frac{T_{h, l}-\mu_{1 l}}{\sqrt{\sigma_{1 l}^{2}}}\right),
$$

and

$$
P_{r}\left\{Y>T_{h, l} \mid b=0, Z \neq w_{l}\right\}=Q\left(\frac{T_{h, l}-\mu_{0 l}}{\sqrt{\sigma_{0 l}^{2}}}\right) .
$$

The function $Q(x)$ is the normalized Gaussian tail probability and can be expressed as

$$
Q(x)=\frac{1}{\sqrt{2 \pi}} \int_{x}^{\infty} e^{-s^{2} / 2} d s .
$$

\section{$5 \quad$ Results and Discussions}

\subsection{Simulink Results}

We have investigated the multimedia transmission in OCDMA computer network using the simulink models and simulation parameters as described in the previous section. The simulated results of the multimedia transmission in OCDMA computer network without any noise (AWGN channel) are as follows.

The spectrum can be monitored using FFT Spectrum Scope2 through Zero Order Hold with sample time of $9.5 \times 10^{-13}$ and FIR Decimation filter at sampling length of 8192 in Figure 5. Signal spectrum of user 1 after encoding process is 
shown in Figure 5, which is monitored after modulation process of user 1's codeword with generated data output of OOK-Modulator1. It can be used to evaluate system performance by observing its optical spectrum.

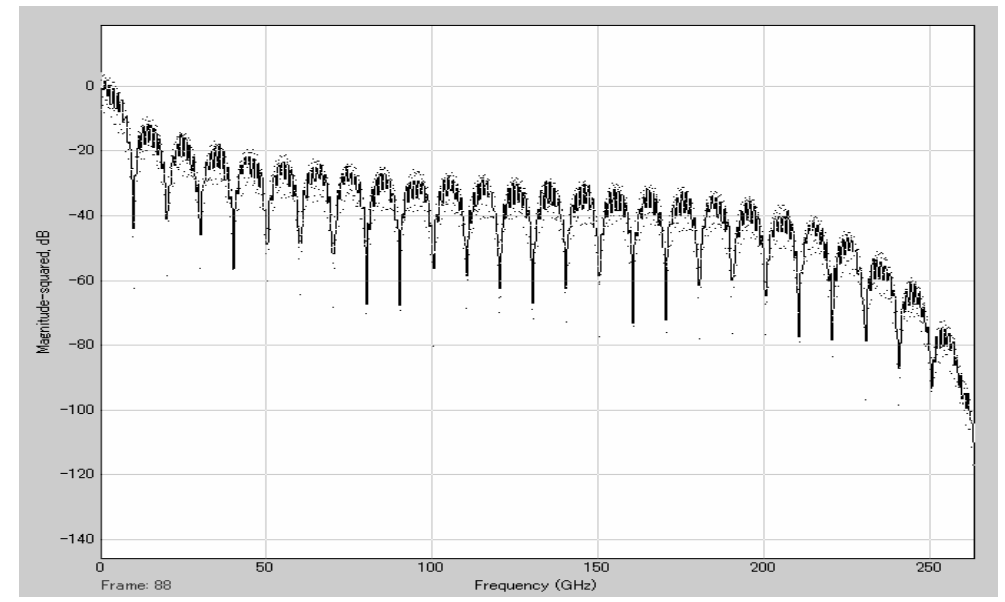

Figure 5 Signal spectrum of user 1 after encoding process.

The spectrum can also be monitored similarly for Figure 6 . The figure shows signal spectrum of user 1 , which is monitored before decoding process or after transmission through star coupler input of Correlation 1 in Figure 3. The spectrum increases and it is different from the encoded spectrum due to the effect of interference from the other users.

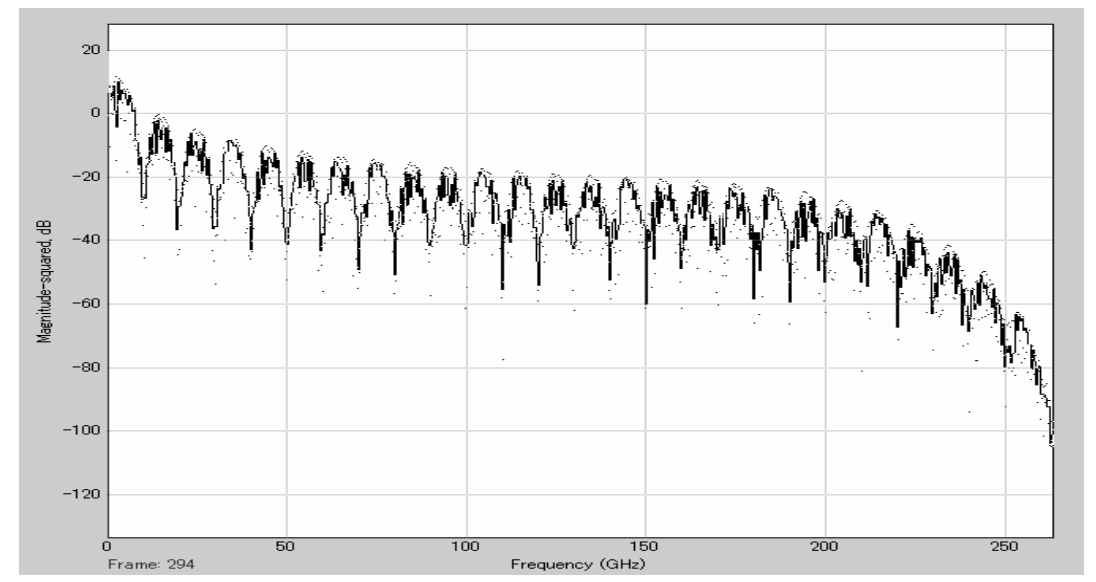

Figure 6 Signal spectrum of user 1 before decoding process. 
Figure 7 shows the transmitted signals of the six-user in the network in the time domain for two different code weights users to accommodate two different services. These output signals are passed through a star coupler. Then, active users superimpose their information signals through the star coupler. The superimposed signals of the six-user are shown in Figure 8. They are then distributed to each receiver. To properly decoding the data, decoder is matched to the desired codeword.

The decoded signals finally arrive at optical receivers. The received signals for the six-user are shown in Figure 9. In the figure, the received signals are different from the transmitted signal because of different frame of the signals. A comparison between the transmitted and received signals has been evaluated for user 1 , where the transmitted and received signals are the same as shown in Figure 10. So the proposed model is a straightforward simulation model to simulate multimedia transmission for OCDMA computer network.

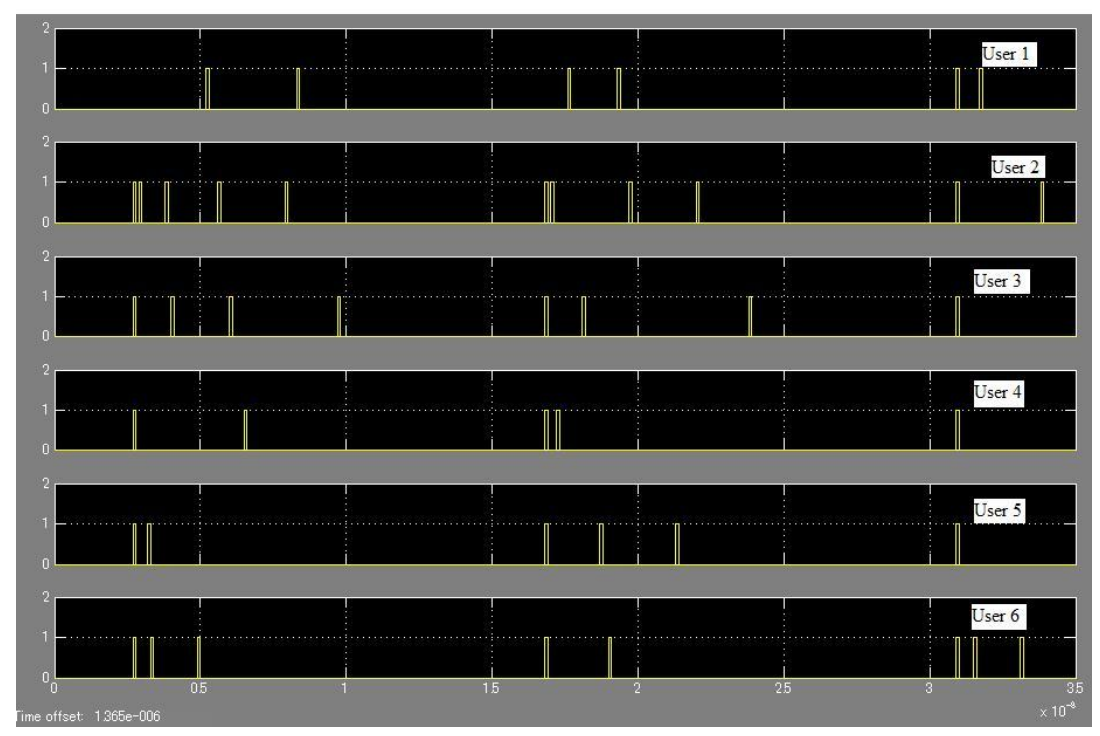

Figure 7 Transmitted signals of six-user in the network. 


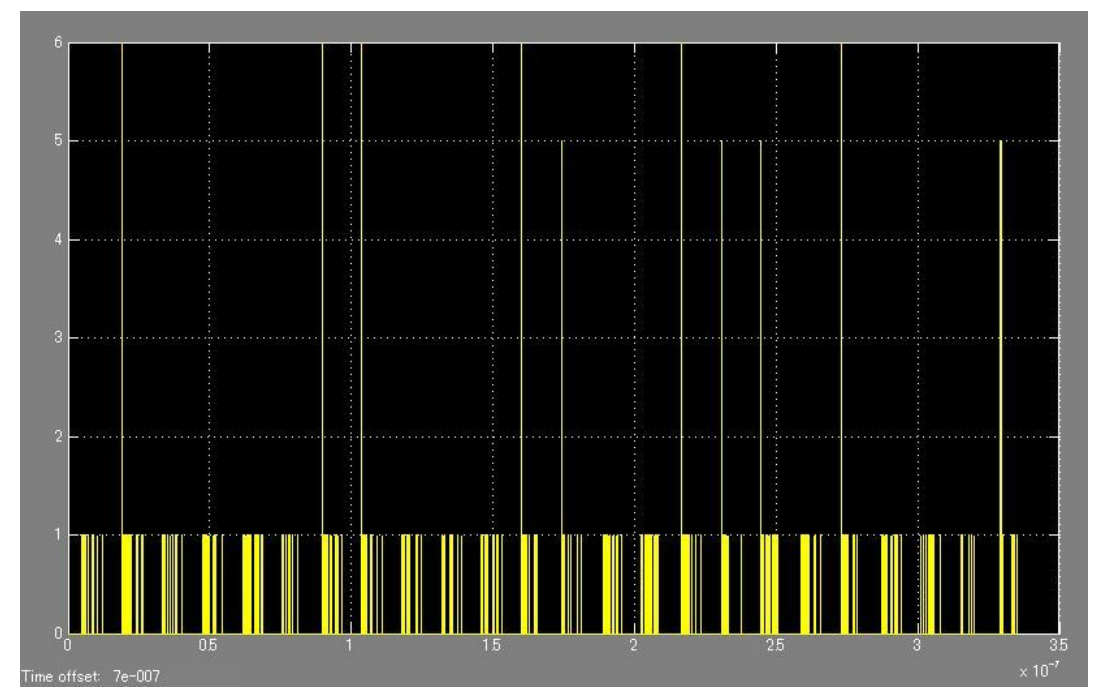

Figure 8 Superimposed signals of six-user in the network.

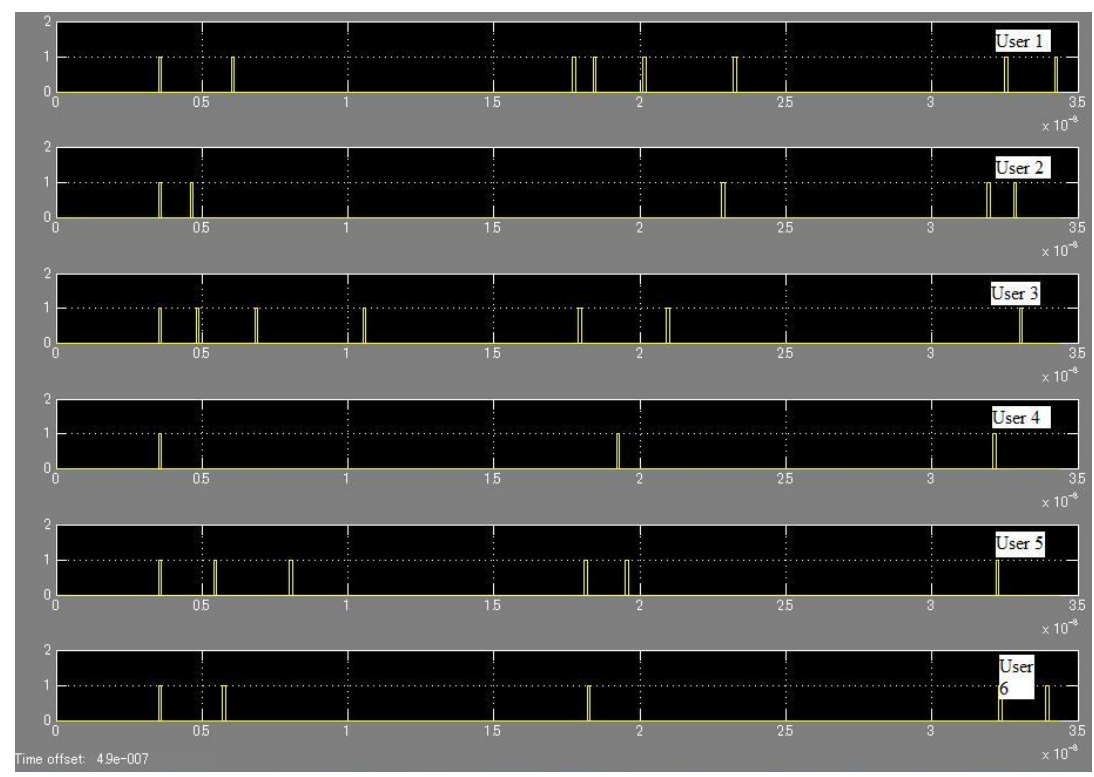

Figure 9 Received signals of six-user in the network. 


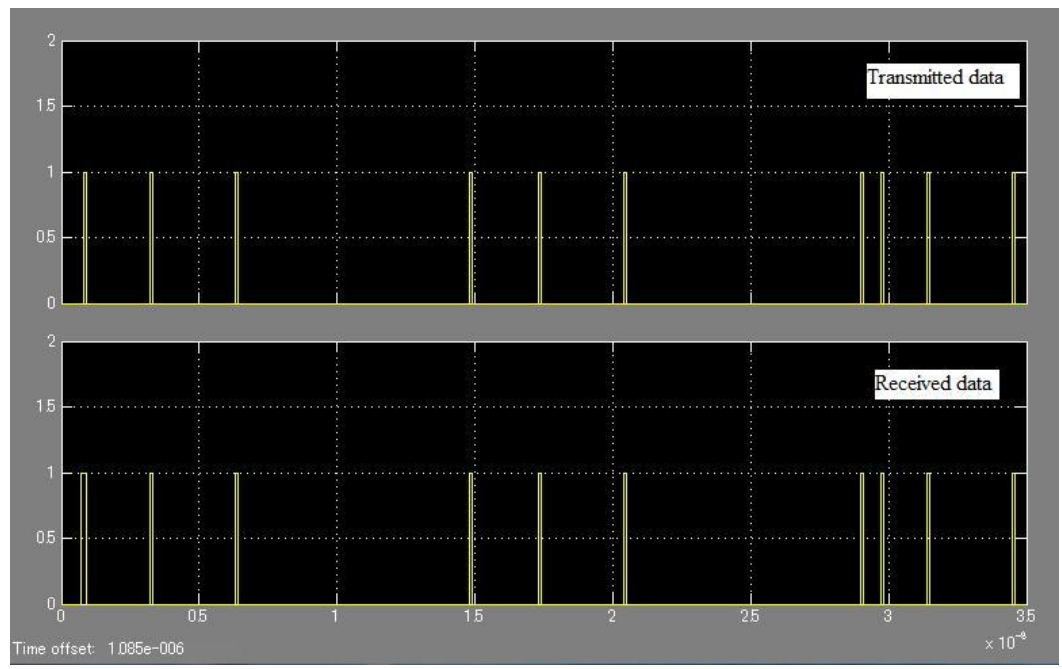

Figure 10 Comparison of transmitted signal and received signal for user 1.

Figure 11 shows binary eye diagrams of the received signal for user 1 and corresponding binary detected signals. Eye diagram is a very successful way of assessing the quality of a digital signal [18]. Therefore, as shown in the eye diagram, we can confirm that it has successfully transmitted the data with OOK modulation in the condition of four-pair users and the $(141,\{5,4\}, 1,\{1 / 2,1 / 2\})$ strict VW-OOC. As indicated by the simulation results, the proposed model without any noise is capable for implementation OCDMA multimedia computer networks.

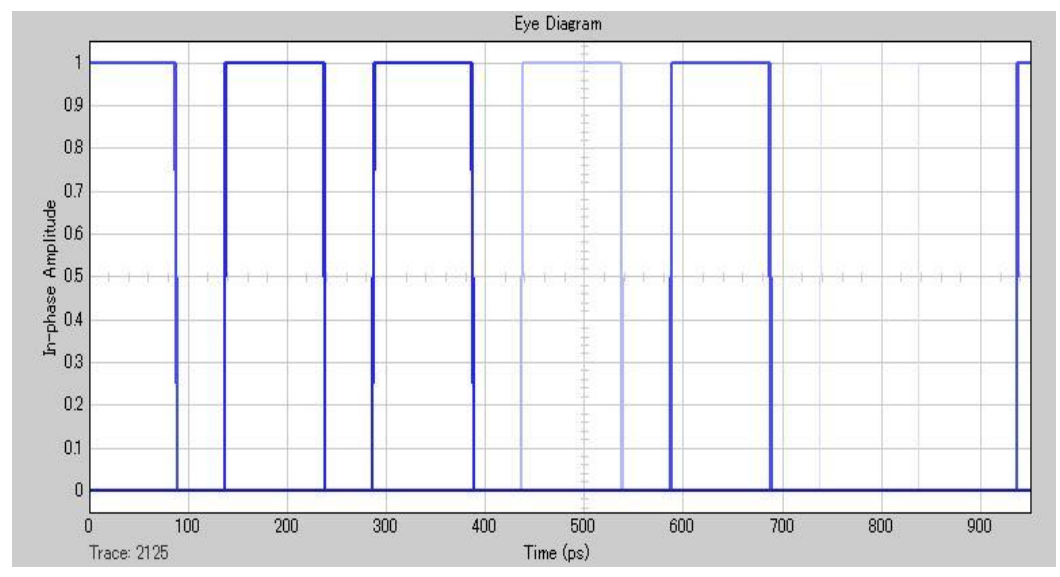

Figure 11 Received eye-diagram for user 1. 


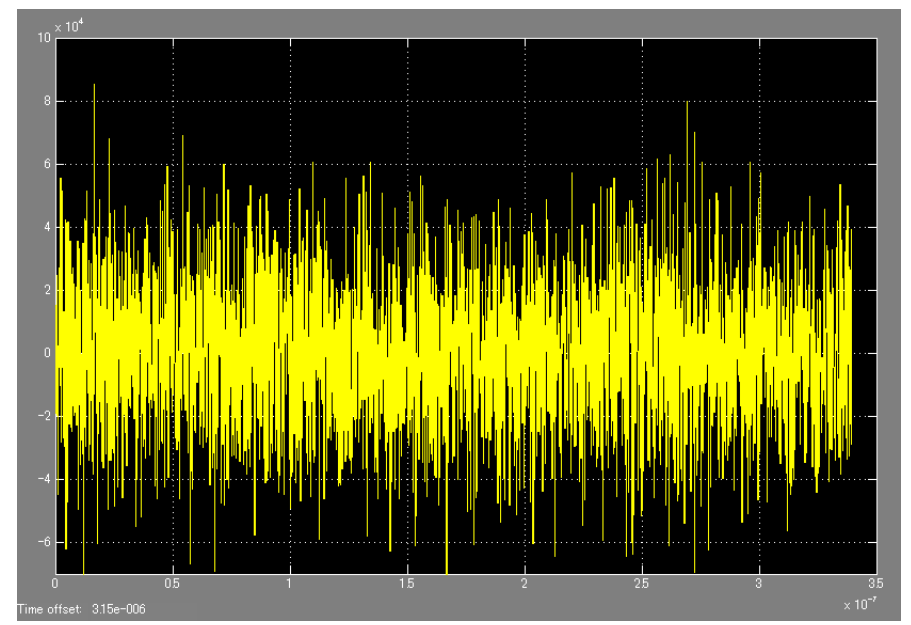

Figure 12 Superimposed signals of six-user in the network with AWGN channel.

The results of simulation model in Figure 4 are almost similar to the results of the simulation model in Figure 3. However, the superimposed signals using AWGN channel are different from the superimposed signal without any noise as shown in Figure 12. Therefore, the effect of noise in practical multimedia transmission of OCDMA computer network should be taken into consideration. We theoretically show the results of effect of noise in OCDMA computer in the next sub section due to no optical noise function in MATLAB/simulink.

\subsection{Noise Analysis Results}

A $(1601,(5,4), 1,(1 / 2,1 / 2))$ strict VW-OOC has been considered in our numerical simulation. In this code set, 50 users with small weight codewords and 50 users with large weight codewords can be gained. The bit error probability in Eqs. (11) and (12) with APD and thermal noise have been evaluated by using the code and network parameters listed in Table 3.

Table 3 Typical network parameters.

\begin{tabular}{lcc}
\hline \multicolumn{1}{c}{ Name } & Symbol & Value \\
\hline Light wavelength & $\lambda_{\mathrm{o}}$ & $1.3 \mu \mathrm{m}$ \\
APD gain & $G$ & 100 \\
APD eff. ionization ratio & $k_{\text {eff }}$ & 0.02 \\
APD quantum efficiency & $H$ & 0.6 \\
APD dark current & $I_{d}$ & $1 \mathrm{nA}$ \\
Received load resistor & $R_{L}$ & $50 \Omega$ \\
Data bit rate & $R_{T}$ & $20 \mathrm{Mb} / \mathrm{s}$ \\
Laser chip width & $T_{c}$ & $0.03 \mathrm{~ns}$ \\
Receiver noise temperature & $T^{\mathrm{o}}$ & $300,6000^{\circ} \mathrm{K}$ \\
\hline
\end{tabular}


The bit error probability versus the average received laser power for the systems with and without optical DHL are shown in Figures 13 and 14, for $M_{1}=M_{2}=$ 50 , and for low and high thermal noise, with noise temperature 300 and $6000^{\circ}$ $\mathrm{K}$, respectively. From Figures 13 and 14, we can see that the bit error probability decreases as the average received laser power increases. This is simply because the effect of noise is not negligible at small received laser power. Since the code contains variable weights, users with large code weight $w_{1}=5$ perform better than those with small code weight $w_{2}=4$. Furthermore, the bit error probability of the receiver with DHL can be better than the receiver without DHL with high received laser power, $\mathrm{P}_{\mathrm{av}} \geq-67.5 \mathrm{dBm}$ in the low thermal noise and $\mathrm{P}_{\mathrm{av}} \geq-60 \mathrm{dBm}$ in the high thermal noise. It may be true because the non-ideal link with high received power would behave as the ideal link. This characteristic is also similar to the performance of system with APD noise and thermal noise by using OOC with constant weight [15]. We also can observe that thermal noise degrades the performance as the noise temperature increases.

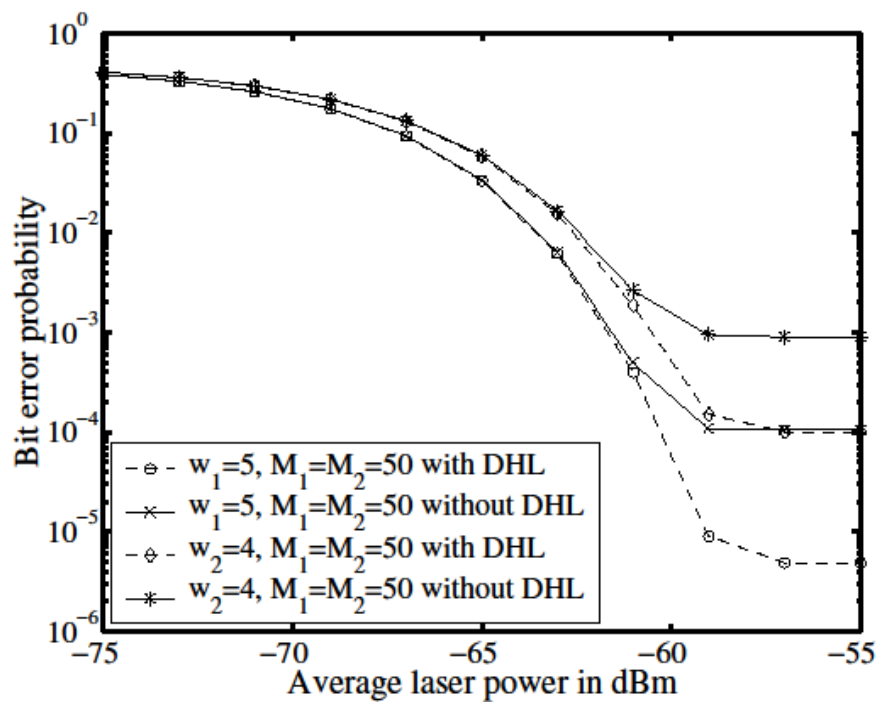

Figure 13 Bit error probability versus the average laser power for $(1601,(5,4), 1,(1 / 2,1 / 2))$ strict VW-OOC, with APD noise and low thermal noise. 


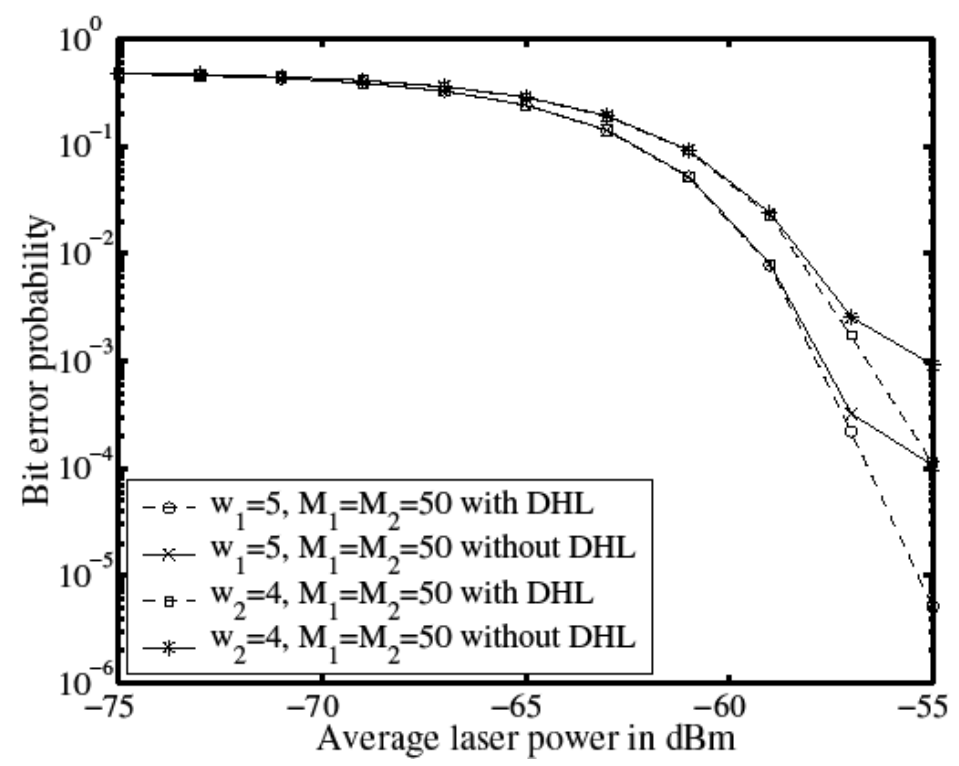

Figure 14 Bit error probability versus the average laser power for $(1601,(5,4), 1,(1 / 2,1 / 2))$ strict VW-OOC, with APD noise and high thermal noise.

\section{Conclusion}

In this paper, we have proposed simulation and noise analysis of multimedia transmission in flexible OCDMA computer networks. OCDMA computer network architecture and optical coding for multimedia transmission have been reviewed. Two simulation models; with and without AWGN channel, of multimedia transmission in OCDMA computer network have been presented. Moreover, APD and thermal noise have been theoretically analyzed in this paper. We have demonstrated various output of spectrum signals and eye diagrams of the proposed models for OCDMA computer networks. The results show that the proposed models are straightforward and easy to implement OCDMA computer networks by using the OCDMA technique as MATLAB simulink model. Therefore, OCDMA computer networks are practically investigated as a future candidate technology to a faster communication and a more efficient use of channel. In addition, the bit error probability for OCDMA computer networks that employs strict VW-OOC with APD noise and thermal noise considerations shows that the system performance depended on the value of code weights, the received laser power, APD noise and thermal noise. Therefore, a careful analysis and design are required towards practical OCDMA computer networks. Future works include evaluating with more suitable simulation parameters to actual OCDMA systems 


\section{References}

[1] Spirit, D.M., Ellis, A.D. \& Barnsley, P.E., Optical Time Division Multiplexing: System and Networks, IEEE Commun. Mag., 32(12), pp. 56-62, Dec. 1994.

[2] Brackett, C.A., Dense Wavelength Division Multiplexing Networks: Principle And Applications, IEEE J. Select. Areas Commun., 8(6), pp. 948-964, Aug. 1990.

[3] Prucnal, P.R., Optical Code Division Multiple Access: Fundamental and Applications, Boca Raton: Taylor \& Francis, 2006.

[4] Stok, A. \& Sargent, E.H., Lighting The Local Area: Optical CodeDivision Multiple Access and Quality Of Service Provisioning, IEEE Network, 14(6), pp. 42-46, Jun. 2000.

[5] Mendez, A.J., Gagliardi, R.M., Feng, H.X.C., Heritage, J.p. \& Morookian, J.M., Strategies for Realizing Optical CDMA for Dense, High-Speed, Long Span, Optical Network Applications, J. of Lightwave Technol., 18(12), pp. 1685-1696, Dec. 2000.

[6] Salehi, J.A., Code Division Multiple-Access Techniques in Optical Fiber Networks-Part I: Fundamental Principles, IEEE Trans. Commun., 37(8), pp.824-833, Aug. 1989.

[7] Chung, F.R.K., Salehi, J.A. \& Wei, V.K., Optical Orthogonal Codes: Design, Analysis, And Application, IEEE Trans. Inf. Theory, 35(3), pp. 595-604, May 1989.

[8] Azizoglu, M., Salehi, J.A. \& Li, Y., Optical CDMA Via Temporal Codes, IEEE Trans. Commun., 40(7), pp. 1162-1170, July 1992.

[9] Zhang, J.G., Design of A Special Family of Optical CDMA Address Codes for Fully Asynchronous Data Communications, IEEE Trans. Commun., 47(7), pp. 967-972, Jul. 1999.

[10] Nasaruddin \& Tsujioka, T., Design of Strict Variable-Weight Optical Orthogonal Codes For Differentiated Quality of Service in Optical CDMA Networks, Computer Network, Elsevier, 52(10), pp. 2077-2086, Jul. 2008.

[11] Nasaruddin \& Tsujioka, T., Random Search Codes and Implementation Model for Optical-CDMA-Based Local Area Networks, In Proc. International Conference on Rural Information and Communication Technology (rICT 2009), pp. 9-14, ITB-Bandung, June 2009.

[12] Nasaruddin \& Tsujioka, T., Multiple-Length Variable-Weight Optical Orthogonal Codes for Multi-Rate Multi-Quality Optical CDMA Systems, in Proc. IEEE Global Telecommunications conference (Globecom 2006), OPN02-1, pp. 1-5, San Fransisco, USA, Nov. 2006.

[13] Nasaruddin \& Tsujioka, T., Multiple-Length Variable-Weight Optical Orthogonal Codes for Supporting Multirate Multimedia Services in 
Optical CDMA Networks, IEICE Trans. Commun., E90-B(8), pp. 19681978, Aug. 2007.

[14] Shalaby, H.M.H., Complexities, Error Probabilities, and Capacities of Optical OOK-CDMA Communication Systems, IEEE Trans. Commun., 50(12), pp. 2009-2017, Dec. 2002.

[15] Kwon, H.H., Optical Orthogonal Code-Division Multiple Access SystemPart I: APD Noise and Thermal Noise, IEEE Trans. Commun., 42(7), pp. 2470-2479, July 1994.

[16] Shalaby, H.M.H., Effect of Thermal Noise and APD Noise on The Performance of OPPM-CDMA Receivers, IEEE J. of Lightwave Technol., 18(7), pp. 905-914, July 2000.

[17] Yang, G.C., Variable-Weight Optical Orthogonal Codes for CDMA Network with Multiple Performance Requirements, IEEE Trans. Commun., 44(1), pp. 47-55, Jan. 1996.

[18] Corish, D., Eye Patterns And Error Rate Analysis In Optical Communications," DIT School of Physics, Yearbook 2005, pp. 3-4, 2005.

[19] Abshire, J.B., Performance of OOK and Low-Order PPM Modulations in Optical Communications when Using APD-Based Receivers, IEEE Trans. Commun., COM-32(10), Oct. 1984. 